\title{
Weak convergence under nonlinearities
}

\author{
DIEGO R. MOREIRA ${ }^{1}$ and EDUARDO V. O. TEIXEIRA ${ }^{2}$ \\ ${ }^{1}$ Departamento de Matemática, Universidade Federal do Ceará, Campus do Pici \\ 60455-760 CE, Brazil \\ ${ }^{2}$ Department of Mathematics, University of Texas at Austin, RLM 9.136 \\ Austin, Texas, 78712-1082 U.S.A.
}

Manuscript received on May 31, 2002; accepted for publication on October 25, 2002; presented by JoÃo LuCAS BARBOSA

\begin{abstract}
In this paper, we prove that if a Nemytskii operator maps $\operatorname{Lp}(\Omega, E)$ into $\operatorname{Lq}(\Omega, F)$, for $p$, q greater than 1, E, F separable Banach spaces and F reflexive, then a sequence that converge weakly and a.e. is sent to a weakly convergent sequence. We give a counterexample proving that if $q=1$ and $p$ is greater than 1 we may not have weak sequential continuity of such operator. However, we prove that if $\mathrm{p}=\mathrm{q}=1$, then a weakly convergent sequence that converges a.e. is mapped into a weakly convergent sequence by a Nemytskii operator. We show an application of the weak continuity of the Nemytskii operators by solving a nonlinear functional equation on $\mathrm{W} 1, \mathrm{p}(\Omega)$, providing the weak continuity of some kind of resolvent operator associated to it and getting a regularity result for such solution.
\end{abstract}

Key words: weak continuity, nonlinearities, Nemytskii operator.

\section{INTRODUCTION}

A very important question in Functional Analysis is how to decide if an operator in a Banach space is weakly continuous. Frequently, we meet this issue in variational problems when we have to check the main assumptions of the classical theorems, especially if we are searching for some kind of compactness results. This question becomes more difficult when we deal with nonlinearities. Among the nonlinear operators, there is an outstanding group called Nemytskii operators. We are interested in the weak sequential continuity of these operators. In order to develop these ideas, we consider the notion of a.e. and weak convergence (a.e.w.) and formulate the problem $\alpha_{p, q}$ where $p, q \geq 1$ to be: Let $f$ be a Caratheodory function and suppose that the Nemytskii operator 
associated to $f$ maps $L^{p}(\Omega, \mathbb{E})$ into $L^{q}(\Omega, \mathbb{F})$. Does $N_{f}$ map a.e.w. convergent sequences into a.e.w. convergent sequences? Our goal in this paper is to study under what conditions the problem $\alpha_{p, q}$ is affirmatively answered.

Our paper is organized as follows. In the section 2 , we treat the heart of the matter. We start providing a uniqueness result of convergence in the a.e.w sense for $L^{p}(\Omega, \mathbb{E})$ spaces (Lemma 1). Afterwards, we construct an example which shows the affirmative answer for the problem $\alpha_{p, 1}$ fails to $p>1$ (Example 2) and we establish the solvability of the problem $\alpha_{p, q}$ under the following assumptions: $q>1$ and reflexivity of $\mathbb{F}$ (Theorem 2). At the end of this section, we prove the counterpart of the example 2 which says that the problem $\alpha_{1,1}$ is solvable on bounded domains (Theorem 4). In the section 3, the last section, we are concerned about studying the solvability on $W^{1, p}(\Omega)$ of the equation

$$
f(x, u(x))-\lambda u(x)=\psi(x)
$$

for $\lambda \in \mathbb{R}$ and $\psi \in W^{1, p}(\Omega)$ given. We also provide conditions to the weak sequential continuity of the resolvent operator $R_{\lambda}=\left(N_{f}-\lambda I\right)^{-1}$ on $W^{1, p}(\Omega)$ and we observe a regularity result for such solutions. In the study of the problems $\alpha_{p, q}$, surprisingly, the cases $q=1$ and $q>1$ have been shown very different. Some of these facts turned out to be known, mainly in particular cases; however not in such a generality. We think it is worthwhile to formulate them in a more general form and make them more available. We believe the ideas developed in this paper may be applied in quite different problems.

\section{WEAK CONTINUITY OF THE NEMYTSKII OPERATOR}

Definition 1. Let $\Omega$ be a domain in $\mathbb{R}^{N}$. Let $\mathbb{E}$ and $\mathbb{F}$ be separable Banach spaces. A function $f: \Omega \times \mathbb{E} \rightarrow \mathbb{F}$ is said to be a Caratheodory function if:

(a) for each fixed $v \in \mathbb{E}$ the function $x \mapsto f(x, v)$ is Lebesgue measurable in $\Omega$;

(b) for almost everywhere fixed $x \in \Omega$ the function $f(x, \cdot): \mathbb{E} \rightarrow \mathbb{F}$ is continuous.

In this case we denote $f \in(C)$. Let $\mathcal{M}(\Omega, \mathbb{E})$ be all measurable functions $u: \Omega \rightarrow \mathbb{E}$. It is easy to prove that if $f \in(C)$ then $f$ defines a mapping $N_{f}: \mathcal{M}(\Omega, \mathbb{E}) \rightarrow \mathcal{M}(\Omega, \mathbb{F})$ by $N_{f}(u)(x):=f(x, u(x))$. This mapping is called the Nemytskii operator associated to $f$. The first result we would like to state is an extention to separable Banach spaces of the remarkable theorem due to Vainberg concerning about the Nemytskii operator theory.

Theorem 1 (Lucchetti and Patrone 1980). Let $\mathbb{E}, \mathbb{F}$ be separable Banach spaces. The Nemytskii operator $N_{f}$ maps $L^{p}(\Omega, \mathbb{E})$ into $L^{q}(\Omega, \mathbb{F}), 1 \leq p, q<+\infty$, if and only if there exist a constant $a>0$ and $b(x) \in L_{+}^{q}(\Omega)$ such that

$$
\|f(x, v)\|_{\mathbb{F}} \leq a \cdot\|v\|_{\mathbb{E}}^{p / q}+b(x)
$$


In this case, the operator $N_{f}$ is continuous and bounded, in the sense that maps bounded sets in bounded sets

DefinITION 2. Let $(X, \mathcal{A}, \mu)$ be a measure space, $\mathcal{F}(X, \mathbb{E})$ a topological vector space of $\mathbb{E}$-valued functions defined on $X$ and $\left(f_{n}\right)_{n \geq 1} \subset \mathcal{F}(X, \mathbb{E})$. We said $f_{n} \rightarrow(f, g)$ a.e.w.(almost everywhere and weakly) in $\mathcal{F}(X, \mathbb{E})$ if $f_{n} \rightarrow f$ a.e. in $X$ and $f_{n} \rightarrow g$ in $\mathcal{F}(X, \mathbb{E})$. If $f=g$ we just say $f_{n} \rightarrow f$ a.e.w. in $\mathcal{F}(X, \mathbb{E})$.

The next Lemma gives a kind of uniqueness of the limit in the above convergence in $L^{p}(X, \mu, \mathbb{E})$ spaces.

Lemma 1 (Moreira 2001, Teixeira 2001). Let $(X, \mathcal{A}, \mu)$ be a $\sigma$-finite measure space and $\left(u_{n}\right)_{n \geq 1} \subset$ $L^{p}(X, \mu, \mathbb{E}), 1 \leq p<+\infty$. Suppose that $u_{n} \rightarrow(u, v)$ a.e.w. in $L^{p}(X, \mu, \mathbb{E})$. Then $u=v$, and therefore $u_{n} \rightarrow$ u a.e.w. in $L^{p}(X, \mu, \mathbb{E})$.

Proof. There exists a sequence $\left\{X_{j}\right\}_{j=1}^{\infty}$ of measurable subsets of $X$ such that:

- $\mu\left(X_{j}\right)<+\infty$ for each $j \geq 1$

- $X=\bigcup_{j=1}^{\infty} X_{j}$

Let $j \geq 1$ be fixed. Given $\varepsilon_{1}=1$, by Egorov's theorem, there is a subset $A_{1}$ of $X_{j}, \mu\left(A_{1}\right)<1$ such that $u_{n} \rightarrow u$ in $L^{\infty}\left(X_{j} \backslash A_{1}, \mathbb{E}\right)$, in particular,

$$
u_{n} \rightarrow u \text { in } L^{p}\left(X_{j} \backslash A_{1}, \mathbb{E}\right)
$$

Thus we have $u=v$ a.e. in $X_{j} \backslash A_{1}$. Taking now $\varepsilon_{2}=\frac{1}{2}$ and applying Egorov's theorem again, we obtain a subset $A_{2}$ of $A_{1}, \mu\left(A_{2}\right)<\frac{1}{2}$ such that $u_{n} \rightarrow u$ in $L^{\infty}\left(A_{1} \backslash A_{2}, \mathbb{E}\right)$ hence $u_{n} \rightarrow u$ in $L^{p}\left(X_{j} \backslash A_{2}, \mathbb{E}\right)$ and therefore, we have $u=v$ a.e. in $X_{j} \backslash A_{2}$. Carry on this process we get a decreasing sequence $\left\{A_{n}\right\}_{n=1}^{\infty}, \mu\left(A_{n}\right)<\frac{1}{n}$ and $u=v$ a.e. in $X_{j} \backslash A_{n}$. Set $A_{X_{j}}=\bigcap_{n=1}^{\infty} A_{n}$. This way $\mu\left(A_{X_{j}}\right)=0$ and $u=v$ a.e. in $X_{j} \backslash A_{X_{j}}$. To finish, we define

$$
\begin{gathered}
B_{j}=\left\{x \in X_{j} ; u(x) \neq v(x)\right\} \\
\{x \in X ; u(x) \neq v(x)\} \subset \bigcup_{j=1}^{\infty} B_{j} \subset \bigcup_{j=1}^{\infty} A_{X_{j}}
\end{gathered}
$$

thus

$$
\mu(\{x \in X ; u(x) \neq v(x)\})=0
$$

This concludes the Lemma.

EXAmPle 1 (Teixeira 2001). Let $1<p<+\infty, m \in \mathbb{N}$ and let $\Omega$ be a domain in $\mathbb{R}^{N}$. Every bounded sequence in $W^{m, p}(\Omega)$ contains a subsequence that converges a.e.w. to some function in $W^{m, p}(\Omega)$. Indeed, suppose $\left(u_{n}\right)_{n \geq 1} \subset W^{m, p}(\Omega),\left\|u_{n}\right\|_{W^{m, p}(\Omega)} \leq C$. Since $W^{m, p}(\Omega)$ is reflexive, 
we can suppose that $u_{n} \rightarrow u \in W^{m, p}(\Omega)$. Let $\left\{\Omega_{j}\right\}_{j=1}^{\infty}$ be a nested sequence of relatively compact open sets exhausting $\Omega$. For all $j \geq 1$ let $v_{j} \in C^{\infty}\left(\mathbb{R}^{N} ; \mathbb{R}\right)$ be a function holding the following properties: $0 \leq v_{j} \leq 1, v_{j} \equiv 1$ in $\Omega_{j}, v_{j} \equiv 0$ in $\mathbb{R}^{N} \backslash \overline{\Omega_{j+1}}$. By Sard's theorem, there exists a regular value $0<a<1$ of $v_{j}$. Let $\widehat{\Omega}_{j}=v_{j}^{-1}(a,+\infty)$. So $\left\{\widehat{\Omega}_{j}\right\}_{j=1}^{\infty}$ is a nested sequence of $C^{\infty}$ relatively compact open sets of $\Omega$. Since the embedding $W^{m, p}\left(\widehat{\Omega}_{j}\right) \hookrightarrow L^{p}\left(\widehat{\Omega}_{j}\right)$ is compact, $u_{n} \rightarrow u$ in $L^{p}\left(\widehat{\Omega}_{j}\right)$; therefore, for each $j \geq 1,\left(u_{n}\right)_{n \geq 1}$ has a subsequence converging a.e. to $u$ in $\widehat{\Omega}_{j}$. Using the Cantor Diagonal Argument, we build a subsequence of $\left(u_{n}\right)_{n \geq 1}$ that converges a.e. in $\Omega$ to $u$.

We are interested in the following problem: When does the Nemytskii operator map a.e.w. convergent sequences into a.e.w. convergent sequences? This question is a way of asking about the weak sequential continuity of the Nemytskii operator. More precisely, our problem is

$$
\left(\alpha_{p, q}\right)\left\{\begin{array}{l}
f \in(C) \\
N_{f} \text { maps } L^{p}(\Omega, \mathbb{E}) \text { into } L^{q}(\Omega, \mathbb{F}) \\
\text { Does } N_{f} \text { map a.e.w. convergent sequences into a.e.w. convergent sequences? }
\end{array}\right.
$$

Of course, a.e. convergent sequences are mapped into a.e. convergent sequences by a Nemytskii operator. Actually, what we want to know is when this class of operator maps a.e.w. convergent sequences into weakly convergent sequences.

It is reasonable to suspect that the problem $\alpha_{p, 1}, 1<p<+\infty$, cannot be affirmatively answered because if it were solvable, we would automatically get, without domain dependence, that the embedding $W^{1, p}(\Omega) \hookrightarrow L^{p}(\Omega)$ would be compact. However, there exist many domains where we have lack of compactness of such an embedding. The next example shows this directly.

EXAmple 2. Let $1<p<+\infty$ and $\Omega$ be a domain in $\mathbb{R}^{N}$. Then answer of the problem $\alpha_{p, 1}$ is negative. Indeed, without lost generality we may assume $0 \in \Omega$. Set $f: \Omega \times \mathbb{R} \rightarrow \mathbb{F}$, $f(x, t)=|t|^{p} \cdot v_{0}$, where $v_{0} \in \mathbb{F} \backslash\{0\}$. The Nemytskii operator $N_{f}$ applies $L^{p}(\Omega)$ into $L^{1}(\Omega, \mathbb{F})$. Set $\left(u_{n}\right)_{n \geq 1} \subset L^{p}(\Omega), u_{n}=\left|B_{n}\right|^{-1 / p} \chi_{B_{n}}$, where $B_{n}=\left\{x \in \mathbb{R}^{N} ;|x|<1 / n\right\}$. Since $u_{n} \rightarrow 0$ a.e. in $\Omega,\left\|u_{n}\right\|_{p}=1$ and $L^{p}(\Omega)$ is reflexive, we may assume $u_{n} \rightarrow 0$ a.e.w. It is easy to check that $N_{f}\left(u_{n}\right) \not 0$ in $L^{1}(\Omega, \mathbb{F})$. In fact, from Hahn-Banach theorem, there exists a $\psi \in \mathbb{F}^{*}$ such that $\psi\left(v_{0}\right)=1$. Define $\Psi \in\left[L^{1}(\Omega, \mathbb{F})\right]^{*}$, setting

$$
\Psi(\xi)=\int_{\Omega} \psi \circ \xi(x) d x .
$$

we obtain

$$
1=\left|B_{n}\right|^{-1} \int_{\Omega} \chi_{B_{n}}(x) d x=\int_{\Omega} \psi\left(N_{f}\left(u_{n}\right)\right)(x) d x=\Psi\left(N_{f}\left(u_{n}\right)\right) \nrightarrow 0
$$

But if we have the presence of the reflexivity, the situation changes and we get the following very useful result. The next theorem is an improvement of the result found in (Moreira 2001, Teixeira 2001). 
Theorem 2 (Moreira 2001, Teixeira 2001). Let $1 \leq p, q<+\infty$ with $q \neq 1$ and $\Omega$ be a domain in $\mathbb{R}^{N}$. If $\mathbb{F}$ is reflexive, then answer of the problem $\alpha_{p, q}$ is affirmative.

Proof. By theorem 1, the Nemytskii operator $N_{f}: L^{p}(\Omega, \mathbb{E}) \rightarrow L^{q}(\Omega, \mathbb{F})$ is a bounded map. Suppose $u_{m} \rightarrow u$ a.e.w. in $L^{p}(\Omega, \mathbb{E})$. Since $\left(u_{m}\right)$ is bounded in $L^{p}(\Omega, \mathbb{E}),\left(N_{f}\left(u_{m}\right)\right)$ is bounded in $L^{q}(\Omega, \mathbb{F})$. By reflexivity, we can extract a subsequence $N_{f}\left(u_{m_{k}}\right) \rightarrow v \in L^{q}(\Omega, \mathbb{F})$. Clearly, $N_{f}\left(u_{m_{k}}\right) \rightarrow N_{f}(u)$ a.e. in $\Omega$. Therefore, by Lemma $1, N_{f}\left(u_{m_{k}}\right) \rightarrow N_{f}(u)$ a.e.w. in $L^{q}(\Omega, \mathbb{F})$. So far, we have proven that if $u_{m} \rightarrow u$ a.e.w. in $L^{p}(\Omega, \mathbb{E})$ there exists a subsequence $\left(u_{m_{k}}\right)$ of $\left(u_{m}\right)$ such that $N_{f}\left(u_{m_{k}}\right) \rightarrow N_{f}(u)$ a.e.w. in $L^{q}(\Omega, \mathbb{F})$. We claim that

$$
N_{f}\left(u_{m}\right) \rightarrow N_{f}(u) \text { a.e.w. in } L^{q}(\Omega, \mathbb{F})
$$

In fact, as we have already observed, we only need to show that $N_{f}\left(u_{m}\right) \rightarrow N_{f}(u)$ in $L^{q}(\Omega, \mathbb{F})$. Suppose, by a contradiction, this is not the case. Thus there is a weak neighborhood $N^{w}\left(N_{f}(u)\right)$ of $N_{f}(u)$ and a subsequence $\left(u_{m_{j}}\right), N_{f}\left(u_{m_{j}}\right) \notin N^{w}\left(N_{f}(u)\right) \forall j \geq 1$. Naturally, $u_{m_{j}} \rightarrow u$ a.e.w., then applying the first step of this proof, we obtain a subsubsequence $\left(u_{m_{j_{k}}}\right)$ of $\left(u_{m_{j}}\right)$, $N_{f}\left(u_{m_{j_{k}}}\right) \rightarrow N_{f}(u)$ a.e.w. in $L^{q}(\Omega, \mathbb{F})$, a contradiction, since $N_{f}\left(u_{m_{j_{k}}}\right) \nrightarrow N_{f}(u)$ because $N_{f}\left(u_{m_{j_{k}}}\right) \notin N^{w}\left(N_{f}(u)\right) \forall k \geq 1$.

COROLlaRY 1. If $\Omega$ is a domain in $\mathbb{R}^{N}$ and $u_{m} \rightarrow u$ a.e.w. in $L^{p}(\Omega), 1<p<+\infty$. Then $u_{m}^{+} \rightarrow u^{+}, u_{m}^{-} \rightarrow u^{-},\left|u_{m}\right| \rightarrow|u|$ all these convergences being in the a.e.w. sense in $L^{p}(\Omega)$.

Corollary 2. If $\Omega$ is a domain in $\mathbb{R}^{N}, m \in \mathbb{N}$ and $N_{f}$ maps the $L^{p}(\Omega)$ into $L^{q}(\Omega), 1<p, q<$ $+\infty$, the operator $N_{f}: W^{m, p}(\Omega) \rightarrow L^{q}(\Omega)$ is weakly sequentially continuous.

COROllaRY 3. Let $\Omega$ be a bounded domain in $\mathbb{R}^{N}$. If $u_{n} \rightarrow$ u a.e.w in $L^{p}(\Omega)$ with $p>1$, then for all $1 \leq q<p, u_{n} \rightarrow u$ in $L^{q}(\Omega)$. Consequently, $W^{1, p}(\Omega)$ is compactly embedded in $L^{q}(\Omega)$, for all $1 \leq q<p$, without any regularity condition on $\partial \Omega$.

Proof. Let us fix $0<\varepsilon<p-1$. Since $L^{p}(\Omega) \hookrightarrow L^{p-\varepsilon}(\Omega)$ we have that $u_{n} \rightarrow u$ in $L^{p-\varepsilon}(\Omega)$. Set $f: \mathbb{R} \rightarrow \mathbb{R}$ given by $f(t)=|t|^{p-\varepsilon}$. $N_{f}$ maps $L^{p}(\Omega)$ into $L^{p / p-\varepsilon}(\Omega)$. From theorem 2 , $f\left(u_{n}\right) \rightarrow f(u)$ in $L^{p / p-\varepsilon}(\Omega)$. In particular

$$
\int_{\Omega}\left|u_{n}\right|^{p-\varepsilon} d x \longrightarrow \int_{\Omega}|u|^{p-\varepsilon} d x
$$

So we have that $u_{n} \rightarrow u$ in $L^{p-\varepsilon}(\Omega)$ and $\left\|u_{n}\right\|_{L^{p-\varepsilon}(\Omega)} \rightarrow\|u\|_{L^{p-\varepsilon}(\Omega)}$. Once $L^{p-\varepsilon}$ is uniformly convex, it implies that $u_{n} \rightarrow u$ in $L^{p-\varepsilon}(\Omega)$. Moreover, using again the fact that $L^{r}(\Omega)$ is continuosly embedded into $L^{s}(\Omega)$ for $r \geq s$, we are able to conclude that $u_{n} \rightarrow u$ in $L^{q}(\Omega)$ for all $q \in[1, p-\varepsilon]$. Letting $\varepsilon \rightarrow 0$, we get the result. The second part of the corollary follows from the first part added with example 1 .

It is worthwhile to stand out that the corollary 3 is sharp. In general, $W^{1, p}(\Omega) \hookrightarrow L^{q}(\Omega)$ for $q \geq p$. This fact can be found in (Adams 1975). 
We remark that weak convergence in $L^{p}(\Omega)$ is not suffice to conclude the thesis of theorem 2. In fact, let $\Omega=\left(0, \frac{\pi}{2}\right), u_{n}(x)=\sin (n x) \rightarrow 0$ in $L^{2}\left(0, \frac{\pi}{2}\right)$, and let $f:\left(0, \frac{\pi}{2}\right) \times \mathbb{R} \rightarrow \mathbb{R}$ be given by: $f(x, s)=s^{+}$. Now we note that: $\left\langle 1, f\left(x, u_{n}(x)\right)\right\rangle_{L^{2}}=\frac{1}{n} \int_{0}^{n \frac{\pi}{2}}(\sin (y))^{+} d y$, so:

$$
\limsup _{n}\left\langle 1, f\left(x, u_{n}(x)\right)\right\rangle_{L^{2}} \geq \lim _{s} \frac{1}{4 s+2} \sum_{k=0}^{s} \int_{2 k \pi}^{(2 k+1) \pi} \sin y d y=\frac{1}{2}
$$

It remains to study the problem $\alpha_{1,1}$. In order to start analyzing this problem, we shall state the general version of Dunford-Pettis theorem, obtained by Talagrand in 1984.

THEOREM 3 (Talagrand 1984). Let $\Omega$ be a bounded domain in $\mathbb{R}^{N}$ and $\mathbb{F}$ be a weak complete Banach space. Let $\mathcal{F} \subset L^{1}(\Omega, \mathbb{F})$ be a bounded convex subset. Then $\mathcal{F}$ is weakly relatively compact, if and only if it satisfies the following two conditions:

1. $\left\{\|\varphi\|_{\mathbb{F}}: \Omega \rightarrow \mathbb{R}: \varphi \in \mathcal{F}\right\} \subset L^{1}(\Omega)$ is weakly precompact;

2. for each sequence $\left(\varphi_{n}\right)$ in $\mathcal{F}$, the set of $x \in \Omega$ such that there is a $k$ for which the sequence $\left(\varphi_{n}\right)_{n \geq k}$ is equivalent to the vector basis of $l^{1}$ has measure zero.

Let us point out that from Dunford-Pettis's theorem, the condition 1 above is equivalent to the equiintegrability of $\left\{\|\varphi\|_{\mathbb{F}}: \varphi \in \mathcal{F}\right\}$. The next theorem is the counterpart of example 2 .

THEOREM 4. Let $\Omega$ be a bounded domain in $\mathbb{R}^{N}$. If $\mathbb{F}$ is reflexive, then the answer of the problem $\alpha_{1,1}$ is affirmative.

Proof. Let $u_{m} \rightarrow u$ a.e.w. in $L^{1}(\Omega, \mathbb{E})$. Defining $u_{0}=u$, by the Eberlein-Smulian theorem (Brito 1998 ) the set $K=\left\{u_{m} ; m \geq 0\right\}$ is weakly compact, since it is weakly sequentially compact. Let us denote $X=\overline{c o}(K)$. From Krein's theorem (Brito 1998) we get that $X$ is weakly compact, thus, in particular theorem 3 says that $X$ is equiintegrable. The equiintegrability means that if $\varepsilon>0$ is given; there exists $\delta_{1}>0$ such that

$$
\int_{A}\|u(x)\|_{\mathbb{E}} d x<\varepsilon \quad \forall A \subset \Omega \text { with }|A|<\delta_{1} \text { and } \forall u \in X
$$

By theorem 1, the Caratheodory function $f$ satisfies the following growth condition:

$$
\|f(x, v)\|_{\mathbb{F}} \leq a\|v\|_{\mathbb{E}}+b(x)
$$

where $a>0$ and $b(x) \in L_{+}^{1}(\Omega)$. Let $Y=\overline{c o}\left(N_{f}(K)\right)$. If $v \in \operatorname{co}\left(N_{f}(K)\right)$, there exist functions $u_{1}, \ldots, u_{n} \in K$ and positive numbers $\lambda_{1}, \ldots, \lambda_{n}$ fufilling $\sum_{j=1}^{n} \lambda_{j}=1$ such that $v=\sum_{j=1}^{n} \lambda_{j} \cdot u_{j}$. In 
this way

$$
\begin{aligned}
\int_{A}\|v(x)\|_{\mathbb{F}} d x=\int_{A}\left\|\sum_{j=1}^{n} \lambda_{j} \cdot N_{f}\left(u_{j}\right)(x)\right\|_{\mathbb{F}} d x & \leq \int_{A} \sum_{j=1}^{n} \lambda_{j}\left\|N_{f}\left(u_{j}\right)(x)\right\|_{\mathbb{F}} d x \\
& \leq a \cdot \int_{A}\left\|\sum_{j=1}^{n} \lambda_{j} \cdot u_{j}(x)\right\|_{\mathbb{E}} d x+\int_{A} b(x) d x \\
& <a \cdot \varepsilon+\int_{A} b(x) d x .
\end{aligned}
$$

Since $\lim _{|A| \rightarrow 0} \int_{A} b(x) d x=0$ there exists $\delta_{2}>0$, such that $|A|<\delta_{2} \Longrightarrow \int_{A} b(x) d x<\varepsilon$; therefore

$$
|A|<\delta=\min \left\{\delta_{1}, \delta_{2}\right\} \Longrightarrow \int_{A}\|v\|_{\mathbb{F}} d x<(a+1) \varepsilon
$$

Thus, we obtain

$$
|A|<\delta=\min \left\{\delta_{1}, \delta_{2}\right\} \Longrightarrow \int_{A}\|v\|_{\mathbb{F}} d x \leq(a+1) \varepsilon \forall v \in Y .
$$

We have just verified the condition 1 of theorem 3, for $Y$. However, by hypothesis, the condition 2 we get for free, since $\mathbb{F}$ being reflexive, it does not contain a copy of $l^{1}$; therefore by theorem 3 , the set $Y$ is weakly compact, and thus so is $N_{f}(K)=\left\{N_{f}\left(u_{m}\right): m \geq 0\right\}$. Using again the EberleinSmulian theorem, we can extract a subsequence $u_{m_{k}}$, such that, $N_{f}\left(u_{m_{k}}\right) \rightarrow v$ in $L^{1}(\Omega, \mathbb{F})$. Since $N_{f}\left(u_{m_{k}}\right) \rightarrow N_{f}(u)$ a.e. in $\Omega$, by the Lemma $1, v=N_{f}(u)$ and then $N_{f}\left(u_{m_{k}}\right) \rightarrow N_{f}(u)$ in $L^{1}(\Omega, \mathbb{F})$. So far, we have proven that if $u_{m} \rightarrow u$ a.e.w in $L^{1}(\Omega, \mathbb{E})$ there exists a subsequence $\left(u_{m_{k}}\right)$ of $\left(u_{m}\right)$ such that $N_{f}\left(u_{m_{k}}\right) \rightarrow N_{f}(u)$ in $L^{1}(\Omega, \mathbb{F})$. We can repeat the same argument used in the proof of theorem 2 and obtain $N_{f}\left(u_{m}\right) \rightarrow N_{f}(u)$ in $L^{1}(\Omega, \mathbb{F})$.

It is interesting to notice that follows immediately from theorem 4 if $u_{n} \rightarrow u$ a.e.w. in $L^{1}(\Omega, \mathbb{F})$ then $u_{n} \rightarrow u$ in the $L^{1}(\Omega, \mathbb{F})$-norm topology.

\section{AN APPLICATION}

We shall provide an application of theorem 2 by solving a general nonlinear equation on the Sobolev spaces $W^{1, p}(\Omega)$. The problem studied here is a very natural question for the Nemytskii operator on Sobolev spaces. Indeed, the problem we shall work on is:

Let $1<p<\infty$, let $\Omega$ be a bounded domain in $\mathbb{R}^{N}$ and let $f: \Omega \times \mathbb{R} \rightarrow \mathbb{R}$ be a Lipschitzian function (In this paper, the Lipschtz norm is defined using the sum norm in euclidean space, i.e, $\left.|(x, s)|_{\mathbb{R}^{N} \times \mathbb{R}}=|x|_{\mathbb{R}^{N}}+|s|_{\mathbb{R}}\right)$, such that

$$
|f(x, s)| \leq a|s|+b(x)
$$


for some $a>0$ and some $b \in L_{+}^{p}(\Omega)$. Given a $\psi \in W^{1, p}(\Omega)$, and given a $\lambda \in \mathbb{R}$ we are interested in finding $u \in W^{1, p}(\Omega)$ such that

$$
f(x, u(x))-\lambda u(x)=\psi(x) \quad \text { a.e. } x \in \Omega
$$

Definition 3. Let $f: \Omega \times \mathbb{E} \rightarrow \mathbb{F}$ be a Caratheodory function such that the Nemytskii operator $N_{f}$ maps $L^{p}(\Omega, \mathbb{E})$ into $L^{q}(\Omega, \mathbb{F})$. We define $\mathcal{T}(f)=\inf \left\{a>0: \exists b \in L_{+}^{q}(\Omega)\right.$ such that $\left.\|f(x, s)\|_{\mathbb{F}} \leq a\|s\|_{\mathbb{E}}^{q / p}+b(x)\right\}$.

Let us remark that the infimum on this definition actually is a minimum. Indeed, let $a_{n}$ be a minimizing sequence for $\mathcal{T}(f)$, and let $b_{n} \in L^{p}(\Omega)$ be functions such that the following inequality $\|f(x, s)\|_{\mathbb{F}} \leq a_{n}\|s\|_{\mathbb{E}}^{q / p}+b_{n}(x)$ holds for all $n \in \mathbb{N}, s \in \mathbb{R}$ and a.e. $x \in \Omega$. Taking the lim inf ${ }_{n}$, we find $\|f(x, s)\|_{\mathbb{F}} \leq \mathcal{T}(f)\|s\|_{\mathbb{E}}^{q / p}+\underline{b}(x)$ where $\underline{b}(x)=\liminf _{n} b_{n}(x)$.

THEOREM 5. The problem above is answered affirmatively for all $\lambda>\|f\|_{\text {Lip }}$. Moreover the solution is unique and the operator $\left(N_{f}-\lambda I d\right)^{-1}: W^{1, p}(\Omega) \rightarrow W^{1, p}(\Omega)$ is sequentially weakly continuous.

Proof. Initially, we remark that $\mathcal{T}(f) \leq\|f\|_{\text {Lip }}$. In fact, since

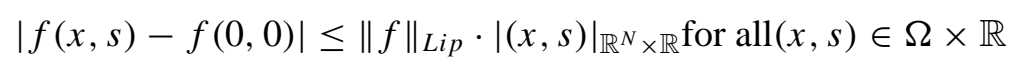

we get

$$
|f(x, s)| \leq\|f\|_{L i p} \cdot|s|+\left\{\|f\|_{L i p} \cdot|x|_{\mathbb{R}^{N}}+|f(0,0)|\right\} .
$$

Let us start by estimating $\|f(x, \xi(x))\|_{W^{1, p}(\Omega)}$ :

$$
\begin{aligned}
\|f(x, \xi(x))\|_{W^{1, p}(\Omega)} & =\|f(x, \xi(x))\|_{L^{p}(\Omega)}+\sum_{j=1}^{N}\left\|D_{j} f(x, \xi(x))\right\|_{L^{p}(\Omega)} \\
& \leq \mathcal{T}(f) \cdot\|\xi\|_{L^{p}(\Omega)}+\|\underline{b}\|_{L^{p}(\Omega)}+\sum_{j=1}^{N}\left\|D_{j} f(x, \xi(x))\right\|_{L^{p}(\Omega)} \\
& \leq \mathcal{T}(f) \cdot\|\xi\|_{L^{p}(\Omega)}+\|\underline{b}\|_{L^{p}(\Omega)}+\|f\|_{L i p} N|\Omega|^{1 / p}+\|f\|_{L i p} \sum_{j=1}^{N}\left\|D_{j} \xi\right\|_{L^{p}(\Omega)} \\
& \leq\|f\|_{L i p} \cdot\|\xi\|_{W^{1, p}(\Omega)}+C(f, N, p, \Omega)
\end{aligned}
$$

This estimative above tell us $N_{f}: W^{1, p}(\Omega) \rightarrow W^{1, p}(\Omega)$ is a bounded operator. Therefore, from the same argument found on the final step of theorem 2, we conclude $N_{f}$ is sequentially weak continuous.

Let us define $\Lambda: W^{1, p}(\Omega) \rightarrow W^{1, p}(\Omega)$ by

$$
\Lambda(\xi)=\frac{1}{\lambda}[f(x, \xi(x))-\psi]
$$


We observe that once $N_{f}$ is sequentially weak continuous, so is $\Lambda$. Moreover, to solve $(P)$ is equivalent to find a fixed point of $\Lambda$.

For all $\xi \in W^{1, p}(\Omega)$

$$
\begin{aligned}
\frac{1}{\lambda}\|f(x, \xi(x))-\psi\|_{W^{1, p}(\Omega)} & \leq \frac{1}{\lambda}\left(\|f(x, \xi(x))\|_{W^{1, p}(\Omega)}+\|\psi\|_{W^{1, p}(\Omega)}\right) \\
& \leq \frac{\|f\|_{L i p}}{\lambda}\|\xi\|_{W^{1, p}(\Omega)}+C(f, N, p, \Omega, \psi)
\end{aligned}
$$

Let us fix $M>\frac{C(f, N, p, \Omega, \psi)}{1-\frac{\|f\|_{L i p}}{\lambda}}$. For such a $M$ we see that if $\|\xi\|_{W^{1, p}(\Omega)}<M$

$$
\|\Lambda(\xi)\|_{W^{1, p}(\Omega)}<\frac{\|f\|_{L i p}}{\lambda} \cdot M+C(f, N, p, \Omega, \psi)<M
$$

In other words, $\Lambda$ maps the ball of radius $M$ in $W^{1, p}(\Omega)$ into itself, i.e., $\Lambda: B_{W^{1, p}}[M] \rightarrow B_{W^{1, p}}[M]$. Let $X$ denote $B_{W^{1, p}}[M]$ endowed with the weak topology. So $X$ is a compact convex subset of a locally convex space. In additional, as we pointed out before, $\Lambda: X \rightarrow X$ is a continuous map.

Finally, we can use the Leray-Schauder-Tychonoff fixed point theorem (Dunford and Schwartz 1964), and conclude that $\Lambda$ has a fixed point which is precisely a solution of $(P)$. Now, let us suppose that there exist $u_{1}, u_{2} \in W^{1, p}(\Omega)$ such that

$$
f\left(x, u_{1}(x)\right)-\lambda u_{1}(x)=\psi(x) \quad \text { and } \quad f\left(x, u_{2}(x)\right)-\lambda u_{2}(x)=\psi(x)
$$

Subtracting these equations we find $f\left(x, u_{1}(x)\right)-f\left(x, u_{2}(x)\right)=\lambda\left(u_{1}(x)-u_{2}(x)\right)$. Therefore

$$
\left|f\left(x, u_{1}(x)\right)-f\left(x, u_{2}(x)\right)\right|=\lambda\left|u_{1}(x)-u_{2}(x)\right| \leq\|f\|_{L i p} \cdot\left|u_{1}(x)-u_{2}(x)\right| .
$$

If $u_{1}(x)-u_{2}(x) \neq 0$, we would be able to cancel this expression at the inequality above and we would find, $\lambda \leq\|f\|_{\text {Lip }}$. Hence the solution of $(P)$ is unique.

In order to study the weak continuity of $\Theta=\left(N_{f}-\lambda I d\right)^{-1}: W^{1, p}(\Omega) \rightarrow W^{1, p}(\Omega)$, we shall use the same idea found in the final step of theorem 2 . Since all weakly convergent sequence in $W^{1, p}(\Omega)$ has a subsequence converging a.e., to prove $\Theta$ is sequentially weakly continuous, it is enough to show that $\Theta$ is bounded. Suppose $\Theta(\psi)=u$,

$$
f(x, u(x))-\lambda u(x)=\psi(x)
$$

Then

$$
\begin{aligned}
\|\psi\|_{W^{1, p}(\Omega)} & \geq \lambda\|u\|_{W^{1, p}(\Omega)}-\left\|N_{f}(u)\right\|_{W^{1, p}(\Omega)} \\
& \geq\left(\lambda-\|f\|_{L i p}\right)\|u\|_{W^{1, p}(\Omega)}-C(f, N, p, \Omega)
\end{aligned}
$$

Writing in a better way,

$$
\|\Theta(\psi)\|_{W^{1, p}(\Omega)} \leq \frac{\|\psi\|_{W^{1, p}(\Omega)}+C(f, N, p, \Omega)}{\lambda-\|f\|_{L i p}}
$$

This estimative shows the operator $\Theta$ is bounded. 
The main information given by theorem 5 is the regularization of the solution. We observe that if we see the map $\Lambda$, defined on the proof of this theorem, as $\Lambda: L^{p}(\Omega) \rightarrow L^{p}(\Omega)$, it is easy to verify that it is a contraction; therefore from the Banach Fixed Point theorem, the problem $(P)$ has, for all $\psi \in L^{p}(\Omega)$, always a unique solution $u \in L^{p}(\Omega)$, provided $\lambda>\|f\|_{L i p}$. The main point of theorem 5 is that $u \in W^{1, p}(\Omega)$ whenever $\psi \in W^{1, p}(\Omega)$.

Let us point out that in a special case when $f(x, s)=A|s|+b(x), b \in \operatorname{Lip}(\Omega)$, we can improve theorem 5, saying that $(P)$ is solvable for all $\lambda>\mathcal{T}(f)$. However we cannot expect to solve $(P)$ if $\lambda \leq \mathcal{T}(f)$ as the following simple situation show us: Let $f: \Omega \times \mathbb{R} \rightarrow \mathbb{R}$ be defined by $f(x, s)=|s|$. In this case, $\mathcal{T}(f)=\|f\|_{L i p}=1$. Suppose $\lambda \leq 1$, then $f(x, u(x))-\lambda u(x)=$ $|u(x)|-\lambda u(x) \geq 0$. Hence if $\psi \in W^{1, p}(\Omega)$, with $\psi(x)<0$, it is impossible to solve the equation $(P)$.

From these comments, a interesting question arises in this problem: What can we say when $\mathcal{T}(f)<\lambda \leq\|f\|_{\text {Lip }}$ ?

\section{ACKNOWLEDGMENTS}

The second author would like to thank professor Haskell Rosenthal (University of Texas - Austin) for bringing the Talagrand's paper to his attention. The authors are grateful for the financial support by CAPES and CNPq, respectively, which are brazilian government agencies that finance the development of science and technology.

\section{RESUMO}

Neste artigo, provamos que se um operador de Nemytskii aplica $\operatorname{Lp}(\Omega, \mathrm{E})$ no $\operatorname{Lq}(\Omega, \mathrm{F})$, para p, q maiores do que 1, E, F espaços de Banach separáveis e F reflexivo, então uma seqüência que converge fracamente e q.t.p. é transformada em uma seqüência fracamente convergente. Fornecemos um contra-exemplo mostrando que se $q=1$ e p é maior do que 1 , podemos não ter continuidade seqüêncial de tal operador. Contudo provamos que se $\mathrm{p}=\mathrm{q}=1$, então seqüências fracamente convergentes que convergem q.t.p. são aplicadas em seqüências fracamente convergentes por um operador de Nemytskii. Mostramos uma aplicação da continuidade fraca dos operadores de Nemytskii resolvendo uma equação funcional não linear no W1,p $(\Omega)$, provando a continuidade fraca de um tipo de operador resolvente associado ao operador de Nemytskii e obtendo um resultado de regularidade de tal solução.

Palavras-chave: continuidade fraca, não linearidades, operador de Nemytskii.

\section{REFERENCES}

Adams RA. 1975. Sobolev Spaces. Academic Press, New York, p. 122-161.

Brito W. 1998. Compacidad debil en espacios de Banach y aplicaciones de un teorema de R.C. James. Universidad de los Andes, Faculdad de Ciencias, Notas de Matematica, Serie Pre-Print, p. 2-42.

Dunford N And Schwartz J. 1964. Linear operator, 2nd ed., Interscience Publishers, New York, p. 456. 
Lucchetti R and Patrone, F. 1980. On Nemytskii's operator and its application to the lower semicontinuity of integral functionals, Indiana Univ Math J 29(5), p. 703-735.

Moreira D. 2001. Soluções positivas de equações elípticas não lineares na presença de expoentes críticos, Universidade Federal do Ceará, Master degree manuscript, p. 12-15.

Talagrand M. 1984. Weak Cauchy sequence in $L^{1}(E)$. Amer J Math 106: 703-724.

TeiXeIra E. 2001. O princípio de concentração e compacidade e aplicações, Universidade Federal do Ceará, Master degree manuscript, p. 36-43. 